\title{
A review on RF based intruder detection using CW Doppler radar
}

\author{
Lynette M. T. ${ }^{1}$, J. M. Kudargi ${ }^{2}$ \\ ${ }^{1,2}$ (EXTC Department, K. J. Somaiya CoE, University of Mumbai, India)
}

\begin{abstract}
In recent years, electromagnetic waves have become the medium of information exchange in free space for various applications ranging from home appliances to industrial products, from research laborites to defense applications, from mobiles to health monitoring systems. The development in the noncontact based intruder detection system via human vital sign using Doppler radar has attracted considerable interest of researchers. Due to the non contact nature and deep penetration level of radio frequencies, the vital sign detection system includes various domain of application, viz. medical, security, etc. this paper presents review of RF based intruder detection using $C W$ Doppler radar. In this paper, vital sign detection techniques are presented.
\end{abstract}

Keywords - CW Doppler radar, heartbeat, intruder, radar receivers, respiration rate.

\section{INTRODUCTION}

The word protection of the belongings leads to the implementation of security system. Various types of security systems are available today with the types varying with respect to the applications. For intruder detection, various types of burglar alarms are introduced today with the advantages of easy availability, cost effectiveness etc. but, these systems also have drawbacks, such as high rate of false alarms, weather effect, animal activity, air movement, machinery noise, vibration, change in temperature. In 1886, Heinrich Hertz discovered the radiation of electromagnetic waves while their propagation. This led to the discovery of radar technology which was used as intruder sensor in WWII. As the advance use of radar technology for various applications increases rapidly, the recent area of research is human vital sign detection system. In case of intruder detector, where conventional alarms has drawbacks in through the wall detection, weather conditions, the RF based vital sign detection system can provide good solution. This is due to the fact that, RF signals can pass through any weather conditions, they can pass through walls. This system is used when continuous monitoring is required. This paper gives an overview of human vital sign detection system.

\section{DOPPLER RADAR THEORY}

When a source generating waves moves relative to an observer, or when an observer moves relative to a source, there is an apparent shift in frequency. If the distance between the observer and the source is increasing, the frequency apparently decreases, whereas the frequency apparently increases if the distance between the observer and the source is decreasing. This relationship is called Doppler Effect (or Doppler Shift) after Austrian Physicist Christian Johann Doppler (1803-1853).[1]

\section{METHODOLOGY}

According to Doppler theory, a constant frequency signal reflected off an object with a periodically varying displacement will result in a reflected signal at the same frequency, but with a time varying phase $\varphi(\mathrm{t}) \cdot[2]$

Doppler radar motion sensing systems typically transmit a continuous wave $(\mathrm{CW})$ electromagnetic signal that is reflected off a target and then demodulated in the receiver.

$R(t) \approx \cos \left[2 \pi f t-\frac{4 \pi d_{0}}{\lambda}-\frac{4 \pi x(t)}{\lambda}\right]$

The received signal consists of the reflected transmitter signal, the Doppler shift $d_{0}$ with time delay related to the target distance, where $\lambda$ is wavelength.[3] A stationary person's chest can be considered as the target of CW radar since it has a periodic movement with no net velocity and hence will therefore receive a signal similar to the transmitted signal, with its phase modulated by the time-varying chest position, as shown in Figure 1. Demodulating the phase will then provide a signal directly proportional to the chest position, which contains information about movement due to heartbeat and respiration, from which heart and respiration rates can be determined. Non-contact heart and respiration monitors have been developed based on this principle [2]. 


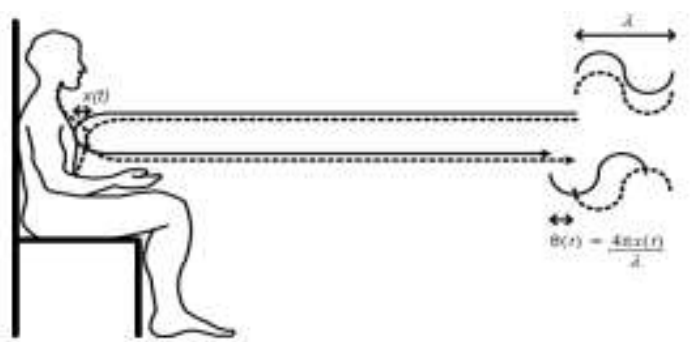

Figure 1: Block diagram of continuous wave radar for measurement of physiological motion. The phase of the reflected signal, $\theta(\mathrm{t})$, is directly proportional to the chest motion, $\mathrm{x}(\mathrm{t})$, and is scaled by the wavelength, $\lambda$.

\section{SYSTEM DESIGN}

The various types of the vital sign monitoring system architecture can be classified on the basis of their receiver design. Receiver used in CW Doppler radar can be direct conversion receiver or quadratuer Doppler receiver. According to the Doppler shift, if the target surface has periodic movement, it is then characterized as phase shift w.r.t to the displacement. Also, if this movement is very small, then at the receiver side, mixer produces an output of low frequency component proportional to the displacement. Fig (2) shows this concept. It is a single channel, direct conversion, $\mathrm{CW}$ radar. A major limitation of the single channel configuration is detection sensitivity to target position due to a periodic phase relationship between the received signal and local oscillator.[1,3]

The received signal can be given as:

$$
R(t)=A_{R} \cos \left[2 \pi f t-\frac{4 \pi d_{0}}{\lambda}-\frac{4 \pi x(t)}{\lambda}+\phi\left(t-\frac{2 d_{0}}{c}\right)+\theta\right]
$$

Received signal which is the RF input to the mixer is

$$
R(t)=A_{R} \cos \left[2 \pi f t-\frac{4 \pi d_{0}}{\lambda}-\frac{4 \pi x(t)}{\lambda}-\frac{4 \pi y(t)}{\lambda}+\phi\left(t-\frac{2 d_{0}}{c}\right)+\theta\right]
$$

Here, $x(t)$ is the signal due to respiration and $y(t)$ is the signal due to the heart beat. LO signal to be given to the mixer is

$$
C(t)=A_{T} \cos (2 \pi f t+\phi(t))
$$

Hence the low frequency signal components obtained after filtering at the output of the mixer is

$$
D(t)=\cos \left[\theta+\frac{4 \pi x(t)}{\lambda}+\Delta \phi(t)\right]
$$

Here, $\Delta \varphi(t)$ is the residual phase noise in the baseband demodulated signal

$$
\Delta \phi(t)=\left[\phi(t)-\phi\left(t-\frac{2 d_{0}}{c}\right)\right]
$$

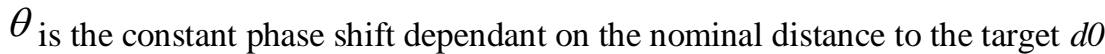

$$
\theta=\left[\frac{4 \pi d_{0}}{\lambda}+\theta_{0}\right]
$$

One major disadvantages of using a homodyne receiver for vital signs monitoring is the amount of DC offset introduced by the system; this makes the signal demodulation difficult. This is due to the vital signal occurs at the low frequency, which is very close to the DC value of the signal.[4]

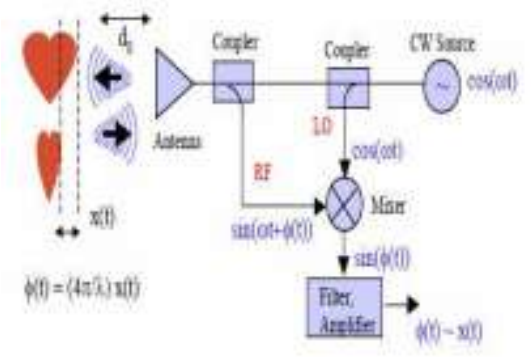

Figure 2: Vital signs remote monitoring Doppler radar system (from $[1,3]$ )

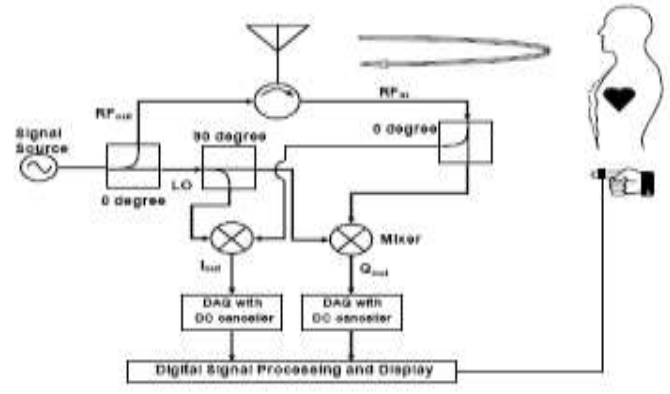

Figure 3: Block diagram of a quadrature Doppler radar system with dc canceller. (from [5]) 
Fig. (3) Shows the quadreture bio radar sensor. This architecture consists of two mixers which provide output on I channel and its $90^{\circ}$ phase shift on Q channel. A channel selector is used to select the optimum output from both the channels. This architecture has advantage of DC cancellation as compared to the single channel receiver.[3] the baseband signal is filtered, amplified and then processed using DSP tools. While quadrature Doppler receiver has more accurate results than direct conversion receiver, the main drawback of this system is large circuitry due to two mixers, phase shifters. Two demodulation methods of quadrature outputs have been proposed: linear (complex) and non-linear (arctangent). Linear demodulation essentially rotates the data to the optimum position, and at lower frequencies, and for small displacements this technique yields accurate results. The arctangent demodulation overcomes the sensitivity to the target position, while also extending the reliable phase deviation detection range that is limited by small angle approximation in single channel receivers.[3] The quadrature baseband output is given as

$$
B(t)=A_{R} \exp [\theta+4 \pi \Delta x(t) / \lambda]
$$

Where $\theta$ is the constant phase shift related to the phase change at the surface of a target and the phase delay between the mixer and antenna.

By arctangent demodulation technique

$$
\begin{gathered}
\phi(t)=\arctan \left(\frac{B_{Q}(t)}{B_{I}(t)}\right) \\
=\arctan \left(\frac{V_{Q}+A_{R} \sin (\theta+4 \pi \Delta x(t) / \lambda)}{V_{I}+A_{R} \cos (\theta+4 \pi \Delta x(t) / \lambda)}\right)
\end{gathered}
$$

Where $V_{Q}$ and $V_{I}$ and refer to the dc offsets of each channel resulting from the finite port to port isolation of the transceiver as well as from clutter reflections. The dc signal contains this dc offset as well as the dc information associated with target's position required for accurate demodulation.[3,5]

\section{RESULTS}

The microwave CW radar vital sign monitoring system can be operated at various frequencies and at various conditions. The higher the operating frequency, the more improved detection sensitivity we obtain.[6,7] The $\mathrm{fft}$ results of the signal obtained from fig. (4) gives the peaks of respiration frequency at $(0.2 \mathrm{~Hz}$ to $0.5 \mathrm{~Hz})$ and the peaks of heartbeat frequency at $0.8 \mathrm{~Hz}$ to $2.5 \mathrm{~Hz}$. Figure below shows the Fast Fourier Transform of the time domain signal obtained.[1]

Fig 5(a) below shows the Doppler output with reference signal taken from finger. It clearly shows that heartbeat and respiration rates can be obtained using Doppler radar architecture. Fig. 5(b) shows the calculated heartbeat and respiration rates obtained from I and Q channels with arctangent and linear demodulation techniques.[3]

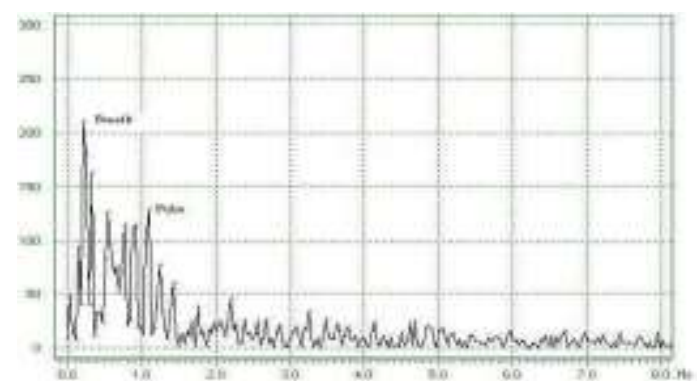

Figure 4: Frequency spectrum of breathing and heartbeat, $\mathrm{D}=1 \mathrm{~m}$ (from [1])

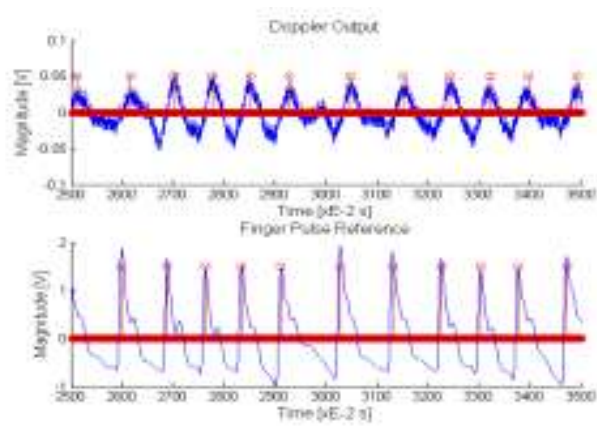

Figure: 5(a)

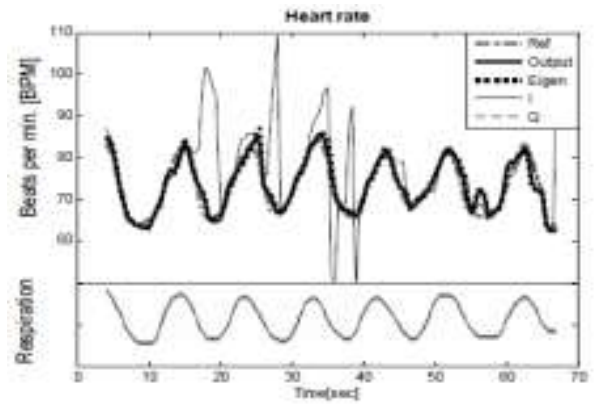

Figure: 5(b) 
Figure 5: Doppler radar output signal (top) and finger pulse reference (bottom) obtained using a quadratuer Doppler radar (a) and extracted heart rate versus time compared to respiratory signal (b) (from [3])

\section{CONCLUSION}

From the review of RF based vital sign detection using CW Doppler radar, it is clearly concluded that, The concept of detecting human vital signs is successfully implemented. It can be stated as quadratuer Doppler receiver gives more accurate results than direct conversion receiver. The drawbacks in direct conversion receiver such as, DC offset can be reduced by the use of quadratuer Doppler receiver. It has the scope of future advancements in various domains for industrial approach such as, clutter cancellation techniques, antenna design with frequency and power constraints, effect on the signal w.r.t. body movement, more than one human are present etc.

\section{References}

[1] Miss Zade Gauri N., Mr. Badnerkar S.S., "A Modern Microwave Life Detection System For Human Being Buried Under Rubble", International Journal Of Advanced Engineering Research And Studies, E-Issn2249 - 8974, Ijaers/Vol. I/ Issue I/October-December, 2011/69-77.

[2] Lohman, B.; Boric-Lubecke, O.; Lubecke, V.M.; Ong, P.W.; Sondhi, M.M.; , "A Digital Signal Processor For Doppler Radar Sensing Of Vital Signs," Engineering In Medicine And Biology Magazine, Ieee, Vol.21, No.5, Pp.161-164, Sept.-Oct. 2002 Doi: 10.1109/Memb.2002.1044188

[3] Olga Boric-Lubecke, Victor M. Lubecke, Isar Mostafanezhad1, Byung-Kwon Park, Wansuree Massagram, Branka Jokanovic, "Doppler Radar Architectures And Signal Processing For Heart Rate Extraction”, Mikrotalasna Revija, Decembar 2009.

[4] Ichapurapu, R.; Jain, S.; John, G.; Monday, T.; Lie, D.Y.C.; Banister, R.; Griswold, J.; , "A 2.4ghz Non-Contact Biosensor System For Continuous Vital-Signs Monitoring," Wireless And Microwave Technology Conference, 2009. Wamicon '09. Ieee 10th Annual, Vol., $\quad$ No., $\quad$ Pp.1-3, $20-21 \quad$ April Doi: 10.1109/Wamicon.2009.5207236.

[5] Byung-Kwon Park, Alex Vergara, Olga Boric-Lubecke, Victor M. Lubecke, Anders Høst-Madsen, "Quadrature Demodulation With Dc Cancellation For A Doppler Radar Motion Detector",

[6] Dany Obeid1, Sawsan Sadek2, Gheorghe Zaharia1, Ghaïs El Zein1, "Feasibility Study For Non-Contact Heartbeat Detection At 2.4 Ghz And 60 Ghz", Xxix General Assembly Of The International Union Of Radio Science (Ursi), Chicago: United States (2008),

[7] Obeid, D.; Sadek, S.; Zaharia, G.; El Zein, G.;, "Touch-Less Heartbeat Detection And Measurement-Based Cardiopulmonary Modeling," Engineering In Medicine And Biology Society (Embc), 2010 Annual International Conference Of The Ieee , Vol., No., Pp.658-661, Aug. 31 2010-Sept.4 2010Doi: 10.1109/Iembs.2010.5627214 